\title{
Análisis de la evapotranspiración potencial en función de elementos climáticos en la zona circunlacustre de la cuenca del Titicaca Peruano
}

\section{Analysis of potential evapotranspiration based on climatic elements in the area circunlacustre of the Titicaca basin of Peru}

\author{
Yury Hamilton Huapaya Cruz \\ IASECON H\&R, Jr. Destua 123, Puno, Perú
}

\begin{abstract}
Resumen
En la zona circunlacustre de la cuenca del Titicaca Peruano se realizó el análisis de la evapotranspiración potencial en función de elementos climáticos, el cual tuvo como objetivos: La determinación de una ecuación regional de la evapotranspiración potencial (Evp) con el método del tanque evaporímetro, calculando el valor del coeficiente(s) del tanque evaporímetro "K(s)", de tal forma se calcule con mayor precisión, facilidad y a menor costo la demanda de agua de los cultivos de importancia económica de dicha zona, evitando los excesos y carencias en la aplicación del agua de riego, estimados por otros métodos de menor precisión; y la descripción del comportamiento de la evapotranspiración potencial de dicha zona. Para tal fin se utilizó la información de las estaciones meteorológicas del SENAMHI de Puno, Huancané, Juli y Desaguadero.

Se tomó los promedios mensuales de los elementos climáticos: Temperatura máxima, mínima, humedad relativa, velocidad del viento, horas de sol y evaporación (Ev) de todos los meses de los años 1981 al 2007. Se determinó la evapotranspiración potencial por el método de Penman Monteith actualizado que es el método de mayor precisión [1], el cual se comparó con la evaporación y posteriormente se determinó K, para cada estación, mes y año respectivo.

Del anterior análisis diremos que para cada estación $\mathrm{K}$, es el promedio de los valores de los Ks de los periodos señalados anteriormente, correspondiendo para Puno 0.78 , Huancané 0.85 , Juli 0.79 y Desaguadero 0.79 . Por lo tanto para la zona circunlacustre $\mathrm{K}$ es 0.80 , este valor se encuentra dentro de los rangos de $\mathrm{K}$ establecidos que varía de 0.40 a 0.85 [2], entonces la ecuación regional que describe la evapotranspiración potencial de la zona circunlacustre es Evp $=0.80^{*} \mathrm{Ev}$.

Los valores estimados de la Evp de cada estación meteorológica se compararon y se graficaron, encontrándose que existe un comportamiento muy similar en cada estación del año guardando estrecha relación en el comportamiento de los vegetales nativos de dicha zona con respecto a su periodo vegetativo y al consumo de agua, lo que indica que la evapotranspiración calculada es coherente con la realidad. Los valores de la evapotranspiración en la zona circunlacustre son 3.55, 2.92, 3.16 y $4.11 \mathrm{~mm} /$ día en el verano, otoño, invierno y primavera respectivamente.
\end{abstract}

Descriptores: Evapotranspiración, coeficiente, meteorología, parámetros, cuenca, circunlacustre.

\begin{abstract}
In the area circunlacustre of the Titicaca's basin, we realized the analysis of potential evapotranspiration based on climatic elements, It had the objectives to: Determining a regional equation of potential evapotranspiration (Evp) with tank evaporation method, calculating the value of tank evaporation coefficient $(\mathrm{K})$, to estimate whit more accurately, easily and at lower cost water demand of crops of economic importance to the area, avoiding excesses and deficiencies in the implementation of water irrigation, estimated by other
\end{abstract}


methods less accurate, and the description of the behavior of potential evapotranspiration that area, for this purpose we used information from the meteorological stations of Puno, Huancané, Juli and Desaguadero.

To this end took the monthly averages of the following climatic elements: Maximum temperature, minimum temperature, relative humidity, wind speed, hours of sun and evaporation of the years 1981 to 2007. The potential evapotranspiration was determined by the method of Penman Monteith update, which is more accurate [2], which was compared with evaporation and subsequently determined $\mathrm{K}$, for each season, month and year in question.

Of the previous analysis we will say, that, for each station $\mathrm{K}$, it is the average of the values of Ks of the periods indicated previously, corresponding for Puno 0.78, for Huancané 0.85, for Juli 0.79 and for Desaguadero 0.79. Therefore in the circunlacustre zone, $\mathrm{K}$ is 0.80 , this value is within the ranges established $\mathrm{K}$ ranging from 0.40 to 0.85 [2], then the regional equation describing the Evp of the circunlacustre zone is Evp $=0.80{ }^{*} \mathrm{Ev}$.

The estimated values of Evp of each weather station were compared and plotted, finding that there is very similar behavior in seasons of the year, correlating with the behavior of plants native to that zone with respect to the vegetative period and to the consumption of water, indicating that evapotranspiration calculation is consistent with the reality. The Evapotranspiration values in the circunlacustre zone are 3.55, 2.92, 3.16 and $4.11 \mathrm{~mm} /$ day in summer, fall, winter and spring respectively.

Keywords: Evapotranspiration, coefficient, meteorology, parameters, basin, circunlacustre.

\section{Introducción}

La evapotranspiración potencial (Evp) es un parámetro que puede ser estimado por diferentes métodos empíricos, siendo los más usados: Penman Monteith, Tanque Evaporímetro, Hargreaves Temperatura, Hargreaves Radiación, Thornthwaite, Blaney y Criddle, entre otros [3]. Todos estos métodos consideran los parámetros climatológicos descritos por coeficientes adimensionales que representan el comportamiento particular de las zonas donde fueron confeccionados dichos métodos; siendo de aplicación universal el de Penman Monteith, por que considera todas las variables que intervienen en la Evp [2]. El método del tanque evaporímetro es la segunda en exactitud, considera la evaporación como básica para la determinación de la Evp, pues en ella intervienen las mismas variables climatológicas de la Evp, acompañado de un coeficiente que varía de 0.40 a 0.85 . [2] y que hasta ahora no fue estimada para las condiciones climáticas del área en estudio.

En el altiplano Puneño se realizó más de 12 estudios de uso consuntivo para diferentes cultivos basados en Lisímetros el cual permite determinar la evapotranspiración real de los cultivos, uno de los estudios comparó los métodos de Lisímetro, radiación, Penman, Hargreaves, Blaney y Criddle, para avena forrajero encontrando que existe diferencia entre ellos [4], el Proyecto Especial Lago Titicaca (PELT) realizó también estudios para bofedales, para avena forrajera y para la alfalfa con los métodos de la FAO y Lisímetro también encontró diferencia entre métodos [5], así mismo se hizo estudios de avena forrajera y alfalfa en el sector de riego Cantería con el método del Lisímetro, Serruto, Hargreaves, Blaney y Criddle existen diferencias entre cada método [6], como se indicó la mayoría de estudios indicaron que existe diferencia significativa entre los diferentes métodos empíricos. Aún no se tiene determinado $\mathrm{K}$ ni se realizó ningún tipo de análisis de la Evp en la zona circunlacustre de la cuenca del Titicaca Peruano.

2001. López \& Dennett, compararon dos métodos de estimación de la Evp: Penman Monteith y del tanque evaporímetro tipo A, para el primer periodo (abr-Jul), obtuvieron entre ambos métodos una correlación de 0.75 significativo estadísticamente, $\mathrm{K}$ vario entre $0.60-0.75$. Para el segundo periodo (jul-nov), obtuvieron entre ambos métodos una correlación de 0,83 significativo estadísticamente, $\mathrm{K}$ vario entre $0.60-0.80$, fue obtenida por el método de la FAO [7].

2005. Martinez, compara la evaporación del tanque tipo "A" con los métodos: Penman, Hargreaves, García-López y Blaney y Criddle, se utilizaron 12 años de registros, se obtuvieron altas 
correlaciones superiores a 0.65 e inferiores a 0.80 [8].

El método de Penman Monteith a pesar de ser el mas exacto, tiene la desventaja de ser muy laborioso en su calculo, requiere la estimación previa de 20 parámetros los cuales son estimados por ecuaciones complejas, y la necesidad de contar con la información de al menos 05 parámetros meteorológicos, para recién aplicar la compleja formula de Penman; además los parámetros meteorológicos tienen un alto costo de adquisición y algunos no son medidos en todas las estaciones meteorológicas de Puno. Por lo tanto es casi inviable su aplicación para los agricultores de la zona circunlacustre. Los otros métodos son menos precisos, ya que los coeficientes y exponentes que las estiman son determinados para zonas específicas.

El presente trabajo de investigación analizó el comportamiento de la evapotranspiración potencial de la zona circunlacustre de la cuenca del Titicaca Peruano y determinó el coeficiente del tanque evaporímetro tipo $\mathrm{A}(\mathrm{k})$ de dicha zona, permitiendo establecer una ecuación regional que describa la Evp de la zona circunlacustre del Titicaca Peruano, para poder estimar con mayor rapidez, facilidad y precisión la evapotranspiración potencial, dando una herramienta práctica, entendible y económica a los agricultores de la zona, permitiéndoles planificar con mayor precisión el riego de tal modo se evite los desperdicios o excesos a la hora de aplicar el agua de riego.

\section{Metodología}

\section{Ámbito de estudio}

El presente trabajo se realizó en la zona circunlacustre de la cuenca del Titicaca de Puno, debido a que en esta zona se cuenta con estaciones meteorológicas que poseen todos los instrumentos necesarios para calcular la Evp por el método de Penman Monteith. Además en esta zona del altiplano se puede desarrollar con mayor intensidad la actividad agrícola, por presentar condiciones apropiadas por el efecto termoregulador del lago Titicaca.
La zona circunlacustre comprende el subtipo climático $A$, clasificados según ONERN 1965 comprendidas entre las altitudes 3812 hasta 3900 msnm. En esta zona se desarrollan cultivos como la papa, quinua, cebada, haba, forrajes, alfalfa, hortalizas entre otros de importancia económica.

\section{Materiales utilizados}

Se tomó los datos de los años de 1981 al 2007 y sus respectivos meses de los siguientes parámetros meteorológicos: Temperatura máxima, temperatura mínima, humedad relativa, velocidad del viento, horas de sol y evaporación (Ev); de las estaciones meteorológicos del SENAMHI de Puno, Huancané, Juli y Desaguadero, las cuales se encuentran distribuidas adecuadamente en la zona circunlacustre del Titicaca Peruano.

Se utilizó el Software Microsoft Excel, SPSS vs.12, 03 millares de hojas entre cuadriculadas, bond y bulki, utensilios de escritorio, un equipo de cómputo con Internet, tesis de uso consuntivo para la revisión bibliográfica.

\section{Tipo de diseño de la investigación}

El presente trabajo de investigación corresponde al diseño no experimental descriptivo de tipo transversal - transaccional correlacional.

Variables independientes: Son la evaporación (Ev) y la evapotranspiración potencial (Evp) calculada por Penman Monteith.

Variable dependiente: Es el coeficiente del tanque evaporímetro tipo A.

\section{Modelo estadístico}

- Se comparó los parámetros climáticos a través del análisis de correlación lineal.

- Para completar datos se hizo el análisis de regresión lineal,

- Para determinar el análisis de homogeneidad se aplicó la prueba de "F" Fisher, para el análisis de saltos en la desviación estándar; y la de "t" Students, para el análisis de saltos en la media, análisis de tendencia en la media y análisis de tendencia en la desviación estándar. 
- Para determinar si el coeficiente del tanque evaporímetro varía de una estación con respecto a otra estación, se hizo el análisis de varianza.

- Para comparar la variación del coeficiente del tanque evaporímetro de una estación con respecto a sus meses se hizo el análisis de varianza.

\section{Procedimiento de cálculo}

A continuación se describe el procedimiento realizado:

1. Se completó los datos faltantes de los elementos climáticos de cada estación meteorológica.

2. Se hizo el análisis de homogeneidad de los datos climatológicos.

3. Se estimó la evapotranspiración potencial por el método de Penman Monteith, utilizando la siguiente expresión:

$$
\mathrm{E} v p=\frac{0.408 \Delta\left(R_{n}-G\right)+\gamma \frac{900}{T+273} u_{2}\left(e_{s}-e_{a}\right)}{\Delta+\gamma\left(1+0.34 u_{2}\right)}
$$

donde:

Evp: Evapotranspiración de referencia [mm día-1],

$R_{n} \quad$ : Radiación neta en la superficie del cultivo [MJ m-2 día-1],

G : Densidad del flujo de calor en el suelo [MJ $\mathrm{m}-2$ día-1],

$\mathrm{T} \quad$ : Temperatura del aire $\left[{ }^{\circ} \mathrm{C}\right]$,

$\mu_{2} \quad$ : Velocidad del viento registrada a $2 \mathrm{~m}$ de altura [m s-1],

$\mathrm{e}_{\mathrm{s}} \quad$ : Presión de vapor de saturación [kPa],

$\mathrm{e}_{\mathrm{a}} \quad$ : Presión de vapor [kPa],

$\mathrm{e}_{\mathrm{s}}-\mathrm{e}_{\mathrm{a}}$ : Déficit de presión de vapor de saturación [kPa],

$\triangle \quad$ : Pendiente de la curva de presión de vapor $\left[\mathrm{kPa}{ }^{\circ} \mathrm{C}-1\right]$,

y $\quad$ : Constante psicrométrica $\left[\mathrm{kPa}{ }^{\circ} \mathrm{C}-1\right]$

4. Se determinó del coeficiente del tanque evaporímetro de todas las estaciones: Con las siguiente relación: K = Evp / Ev. Los valores obtenidos fueron para el mes y año correspondiente de cada parámetro climático analizado.

5. Se realizo el Análisis de Varianza (ANVA) del coeficiente del tanque evaporímetro entre los meses de cada estación. Se tomaron los valores de $\mathrm{K}$ calculados. Cabe indicar que el ANVA fue calculado en el SPSS Vs12 y el Microsoft Excel.

6. Se comparó las series históricas de la evapotranspiración y evaporación:

7. Se determinó el $\mathrm{K}$ de cada estación, se hizo considerando el anterior análisis, es decir se promedió los Ks del periodo que no presenta diferencia significativa.

8. Se hizo el análisis de varianza entre las estaciones meteorológicas.

9. Se determinó el $\mathrm{K}$ de la zona circunlacustre: Se determinó el promedio de todo los Ks de todas las estaciones, tomando los Ks de cada estación, correspondientes al periodo que no existe diferencia significativa de los Ks de dicha estación.

10. Se definió la Evp con una ecuación regional, el cual permite determinar la Evp en función de la evaporación y el $\mathrm{K}$ determinado para la zona circunlacustre Peruano.

11. Se analizó y comparó el comportamiento de la Evp de toda la serie cronológica (1981-2007) de las estaciones meteorológicas por estaciones del año (verano, otoño, invierno, primavera), en un gráfico cuya ordenada es la Evp y la abscisa es las estaciones del año. Los valores para cada estación del año se tomó promediando la Evp de los 3 meses que le corresponden.

\section{Resultados y discusión Análisis de series históricas}

En las Fig.s 1 al 4 se presentan las series históricas de la evaporación de las 4 estaciones de estudio, cuyas características se describen a continuación:

- La tendencia secular es horizontal.

- Los ciclos se repiten año tras año, por lo que tienen una longitud de un año.

- La característica estacional muestra que los valores más altos se dan alrededor del mes de enero y los valores más bajos alrededor del mes de Julio.

Los movimientos irregulares, se dan debido a que la evaporación es un valor muy variable, ya que depende de las características de los otros elementos climáticos mencionados. 
Las cuatro estaciones muestran una irregularidad en sus ciclos, debido a que los valores medidos fueron alterados por los factores externos tales como las precipitaciones y un mal manejo de los operarios que realizan las mediciones, los cuales eran cambiados constantemente por diversos motivos.

Las irregularidades de series históricas de la evaporación también se dieron por la presencia de aves, los cuales beben el agua del tanque, sean reportado aves muertas dentro de los tanques atrapadas en las mallas.

- Para escoger los periodos consistentes de evaporación se compararon con la evapotranspiración potencial visualmente y con el análisis de varianza a través del coeficiente del tanque tipo $\mathrm{A}$.

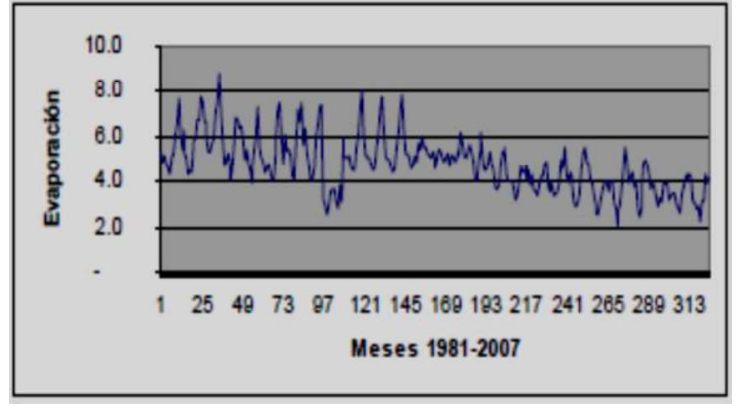

Fig. 1: Serie de evaporación de Puno

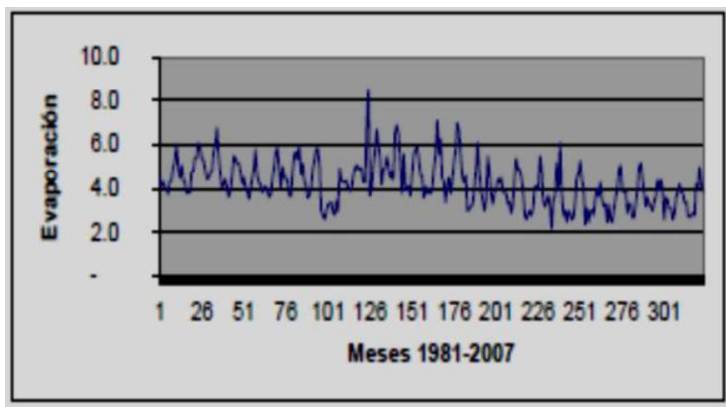

Fig. 2: Serie de evaporación de Huancané

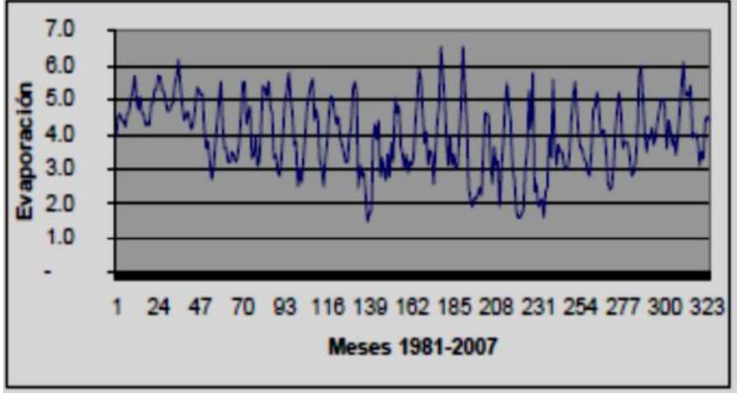

Fig. 3: Serie de evaporación de Juli

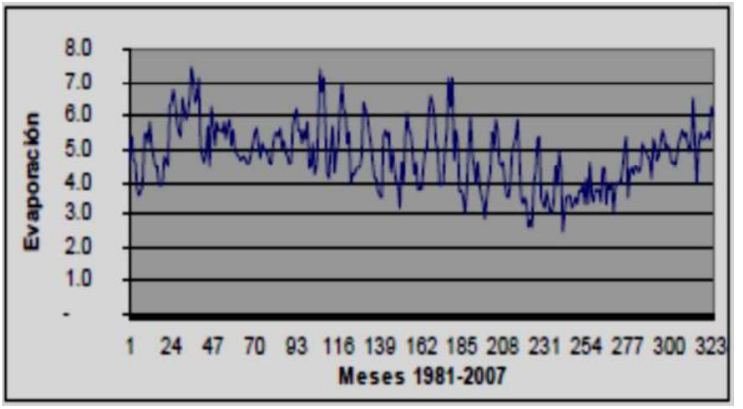

Fig. 4: Evaporación de Desaguadero

En las Fig.s 5 al 8 se presentan las series históricas de la Evp de las 4 estaciones de estudio cuyas características más comunes son las siguientes:

- La tendencia secular es horizontal.

- Los ciclos se repiten año tras año, por lo que tienen una longitud de un año.

La característica estacional muestra que los valores más altos se dan alrededor del mes de enero y los valores más bajos alrededor del mes de julio, en relación directa con la evaporación, por lo que, es factible encontrar un solo valor del coeficiente del tanque evaporímetro.

Se observa que los valores varían de 2.4 a $5 \mathrm{~mm} /$ día, presentándose los valores más altos en verano y los valores más bajo en invierno.

Existe fuerte influencia de la humedad relativa en el comportamiento de la Evp, una cantidad alta de humedad en el aire hace que decrezca la Evp. 


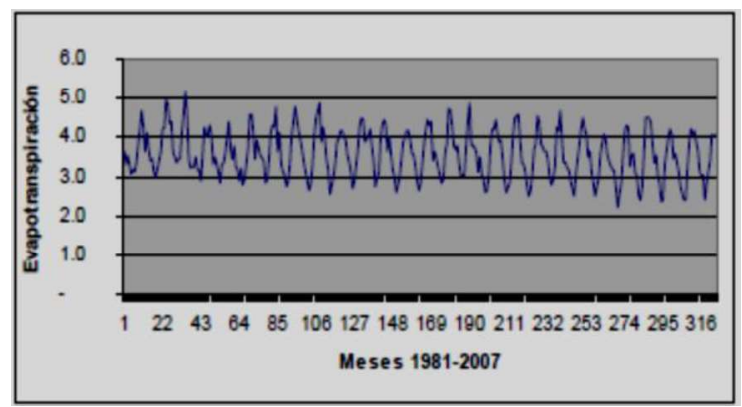

Fig. 5: Serie de Evp de Puno

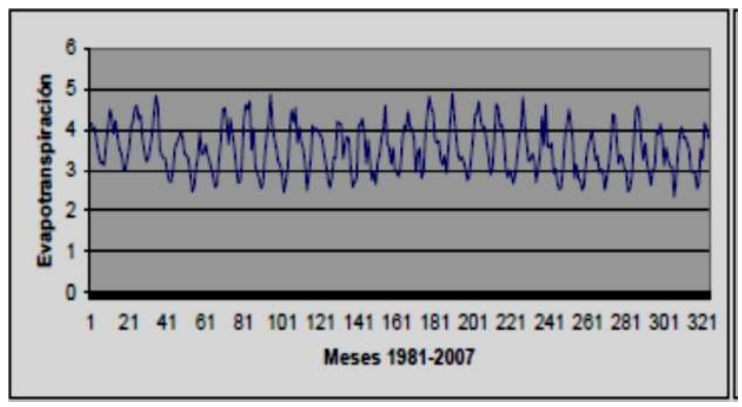

Fig. 6: Serie de Evp de Huancané

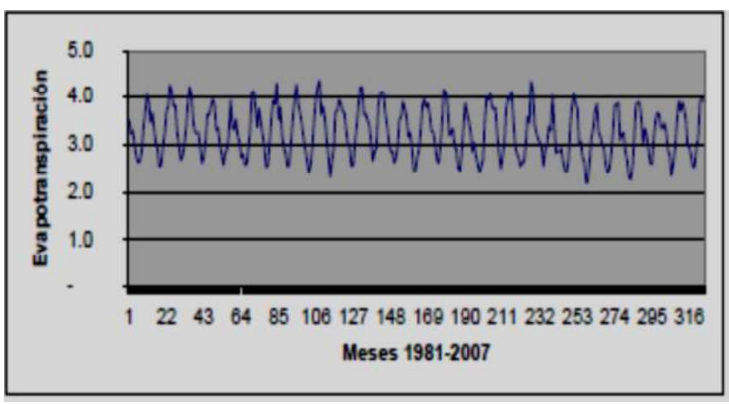

Fig. 7: Serie de Evp de Juli

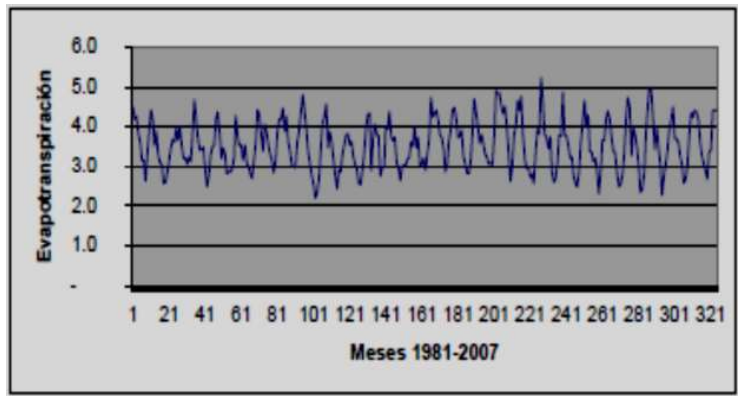

Fig. 8: Serie de Evp de Desaguadero

Determinación de la evapotranspiración potencial (Evp)

En los histogramas de la Evp generados se muestra la uniformidad de los valores de la evapotranspiración, a pesar de la gran variabilidad de la velocidad de viento en las 4 estaciones, la evapotranspiración es homogénea, demostrándose que la humedad relativa, tiene mayor incidencia en el cálculo de la evapotranspiración potencial estimado por el método de Penman Monteith.

La tabla 1 muestra los valores máximos, mínimos y la media de la Evp de las 4 estaciones meteorológicas, dichos valores son iguales estadísticamente entre estaciones lo que indica que la zona circunlacustre tiene un mismo comportamiento climático, indicado que la separación entre estaciones es de $70 \mathrm{~km}$ aproximadamente.

Tabla 1: Valores máximos, mínimos y media de la Evp de las estaciones meteorológicas de los años 1981 al 2007 (mm/día)

\begin{tabular}{|c|c|c|c|}
\hline Estaciones & Media & $\begin{array}{l}\text { Valor } \\
\text { máximo }\end{array}$ & $\begin{array}{l}\text { Valor } \\
\text { mínimo }\end{array}$ \\
\hline Puno & $\underline{3.55}$ & $\underline{5.14}$ & $\underline{2.34}$ \\
\hline Huancané & $\underline{3.42}$ & $\underline{4.88}$ & $\underline{2.06}$ \\
\hline Juli & $\underline{3.26}$ & 4.36 & 2.18 \\
\hline Desaguadero & $\underline{3.52}$ & $\underline{5.22}$ & $\underline{2.16}$ \\
\hline
\end{tabular}

\section{Determinación del coeficiente del tanque evaporímetro}

Estación Puno.- Los valores del coeficiente del tanque evaporímetro "K"de los años 1981-2007, no existe diferencia significativa tienen una desviación estándar de 0.16 y una media de 0.78 , por lo que se considera los valores de $\mathrm{K}$ del periodo 19902007 de esta estación para la comparación entre estaciones.

Estación Huancané.- En el análisis de varianza (1981-2007) se estimo que existe diferencia significativa entre los valores de los $\mathrm{Ks}$, por lo tanto se identificó los siguientes periodos comparando los histogramas de la evaporación y Evp de dicha estación: 1er período de 1981 a 1991, 2do período de 1992 al 2001 y 3er periodo del 2002 al 2007. Siendo el segundo periodo el que no presenta 
diferencia significativa, dicho periodo será tomado para el análisis entre estaciones. Los períodos del 2002 al 2007, no presenta consistencia debido a que en estos años se vino incrementando la población de Huancané, produciendo construcciones cercanas a la estación.

Estación Juli.- En el análisis de varianza (19812007) se obtuvo existe diferencia significativa. Por lo que se identifica los siguientes periodos: 1er periodo de 1985 a 1992, 2do período de 1993 al 2000 y 3er período del 2001 al 2007. Siendo el tercer periodo de 2001 - 2007 que no presenta diferencia significativa.

Estación Desaguadero.- En el análisis de varianza (1981-2007) se obtuvo existe diferencia significativa. Por lo que se identifica los siguientes periodos: 1er periodo de 1981 a 1988, 2do periodo de 1989 al 2001 y 3er periodo del 2002 al 2007. Siendo el 2do periodo de 1989 - 2001 que no presenta diferencia significativa.

A pesar de que se homogenizo la serie histórica de la evaporación en base al periodo correcto, con la fórmula de corrección del análisis de homogeneidad estadística, no varia los resultados obtenidos debido a que dicha ecuación corrige el periodo erróneo, en base a la media y desviación, colocándolo en un intervalo de valores de evaporación hipotético que no es real, por lo que, no lo relaciona proporcionalmente con la evapotranspiración potencial.

Para determinar $\mathrm{K}$ de la zona circunlacustre de la cuenca del Titicaca se toma los promedios anuales de los periodos correctos, de cada estación meteorológica estudiada, ubicando los valores en su periodo respectivo. En el análisis de varianza de las 4 estaciones de $\mathrm{K}$ correspondientes a los años 1990-2007, no existe diferencia significativa a un a de 0.05 y 0.01 . Por lo que el coeficiente del tanque evaporímetro de la zona circunlacustre es 0.80 que es promedio de los periodos escogidos de todas las estaciones (1990 - 2007). El valor de $\mathrm{K}=0.80$ indica que la Evp es el $80 \%$ de la evaporación en la zona circunlacustre del Titicaca Peruano, cuya ecuación regional de dicha zona es Evp = $0.80 *$ Ev.

Análisis de la Evp en la zona circunlacustre
Tal como se observa en la Fig. 9 los valores más altos de la Evp se dan en la primavera, concordando con la característica de esta estación, donde se presenta la mayoría de las plantas en su fase de desarrollo y mayor consumo de agua. Los valores más bajos de la Evp se dan en otoño donde efectivamente no hay cubierta vegetal y la mayoría de las plantas se encuentran en su última fase.

El comportamiento de la Evp en las estaciones Puno, Huancané, Juli y Desaguadero varia en la misma proporción a lo largo del año, por lo que las líneas formadas por cada estación meteorológica son casi paralelas.

Gráficamente se corrobora las conclusiones de algunas investigaciones, que existe diferencia significativa de la Evp entre estaciones del año [9].

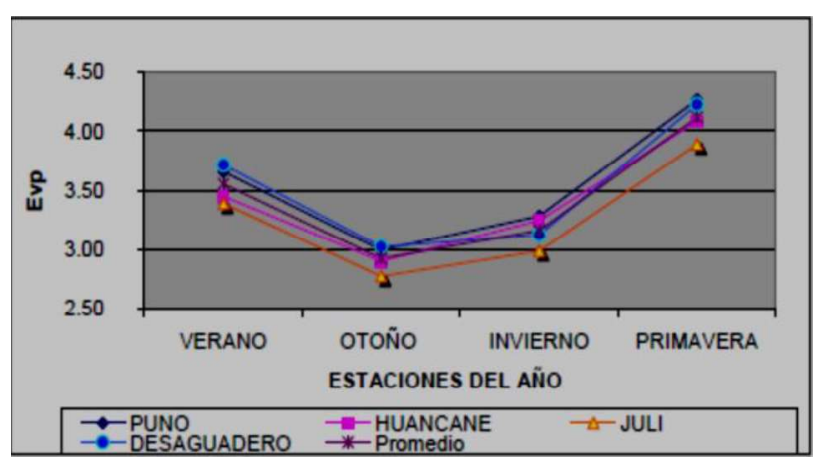

Fig. 9: Promedio de la Evp (1981-2007)

\section{Conclusiones}

La evapotranspiración calculado en la zona circunlacustre promedio es $3.44 \mathrm{~mm} / \mathrm{dia}$, en las 4 estaciones meteorológicas varia en la misma proporción a lo largo del año y describe un comportamiento en estrecha relación con el desarrollo de las plantas nativas y con la campaña agrícola, demostrándose que los cultivos consumirán más agua en primavera y verano, debido que en dichos periodos están en su fase de desarrollo y existe un clima caluroso que colleva a una mayor evapotranspiración en los cultivos. Por lo tanto estos periodos son adecuados para la agricultura. Lo contrario a lo que ocurre con los periodos de invierno y otoño donde la evapotranspiración es menor. Por lo que en dichos periodos no se presentan vegetación en el 
altiplano Puneño, esencialmente por la falta de agua y el exceso de frió.

El coeficiente del tanque evaporímetro de la zona circunlacustre es igual a 0.80 , por lo tanto la ecuación regional que describe la evapotranspiración potencial en función de la evaporación es: $\quad$ Evp $=0.80^{\star} E v$

\section{Agradecimiento}

Agradezco al SENAMHI Puno institución, quien a través del Ingeniero Hérman Zenón Saavedra Aguilar me proporcionaron los datos meteorológicos para realizar la presente investigación, así también al director de la investigación In German Belizario.

\section{Referencias}

[1] F. Yagues, Técnicas de Riego, Mundi Prensa, España, 1996.

[2] G. R. Allend, Evapotranspiración de cultivo, Estudio de la FAO. Riego y Drenaje, Boletín $N^{\circ}$ 56, Italia, 2006.
[3] J. Doorembos, W. D. Pruit, La necesidad de agua de los cultivos, Estudio de la FAO. Riego y Drenaje, Boletín № 24. EEUU, 1976.

[4] C. Chambilla, Requerimiento de agua en el cultivo de avena forrajera, UNA-PUNO, Perú, 1978-1979.

[5] PELT, Avances en la obtención de los coeficientes de cultivo $\mathrm{Kc}$ en el altiplano, Perú, 1999.

[6] S. Olivera, Determinación de uso consuntivo del cultivo de Avena y Alfalfa en el sector de riego Cantera, Universidad Nacional del Altiplano, Venezuela, 2001.

[7] J. López, M. Denté, Comparación de dos métodos para estimar la evapotranspiración de referencia en una zona semiárida de Venezuela, Universidad Occidental Lisandro Alvardo, Venezuela, 2001.

[8] R. Martinez, A. Boueri, P. Escalona, Correlación entre la evaporación de Tina y la evapotranspiración en cinco estaciones climatológicas de Venezuela, Biagro, Venezuela, 2005.

[9] G. F. Ortega, A, Determinación de la Evapotranspiración y balance hidrológico, UNA-PUNO, Perú, 1974.

E-mail: yury_hamilton@hotmail.com 